\title{
Conversation Club: A Promising Practice in Youth Mentoring of Migrants and Refugees
}

\author{
Julia Pryce \\ Michael S. Kelly \\ Mary Lawinger \\ Anne Wildman
}

\begin{abstract}
This paper evaluates Conversation Club, a Canadian after-school group mentoring intervention focusing on the expansion of the program across three separate regions of Ontario. The authors use a multiple methods design, including questionnaires $(n=101)$, post-session process data, and qualitative interviews $(n=18)$, to evaluate how Conversation Club impacts members' feelings of hope, belonging, sense of ethnic identity, and social support. A focus group $(n=7)$ with program facilitators was also conducted to explore the process of dissemination of the Club across regions. Findings suggest that Conversation Club holds promise for newcomer youth across settings. Quantitative data showed significant change $(p<.01)$ in levels of hope and sense of belonging. Interviews revealed an increased sense of belonging, possibility, and social support, as well as improved confidence in communicating with others. Insights regarding use of the Club manual suggest the importance of integrating Conversation Club values with flexibility in facilitation to incorporate the strengths and opportunities of context across regions. Study limitations, as well as implications for further social work research and dissemination of best practices in services for migrant and refugee youth, are discussed.
\end{abstract}

Keywords: Migrant and refugee youth, group mentoring, adolescents

Addressing the international flow of immigrants and refugees is a major concern for governments around the world. In recent years, instability in the Middle East and Northern Africa has caused a migration crisis (Metcalfe-Hough, 2015), with many people fleeing their native countries to avoid violence, as well as to seek improved economic opportunities. In 2015, there were 244 million international migrants, over 16 million of whom were refugees (Migration Policy Institute, 2015).

Canada in particular has emerged as a country willing to accept immigrants and refugees while striving to provide resources to ease the cultural shock and isolation that can often accompany migration (Kantor \& Einhorn, 2016; Vieira, 2015). One in five people living in Canada is foreign-born, and in 2018, Canada plans to receive 310,000 additional immigrants (Government of Canada, 2017; Statistics Canada, 2011). In 2016, people from Syria were the third largest source of immigrants to Canada, making up $11.8 \%$ of the total immigrant population (Government of Canada, 2016; The Canadian Magazine of Immigration, 2017). Immigrants moving from India to Canada, the second largest group, increased by 1\% from 2015 to 2016 (Government of Canada, 2016; The Canadian Magazine of Immigration, 2017). Migrants coming from Democratic Republic of Congo,

\footnotetext{
Julia Pryce PhD, LCSW is an Associate Professor, School of Social Work, Loyola University Chicago, Chicago, IL 60611. Michael Kelly, PhD is a Professor, School of Social Work, Loyola University Chicago, Chicago, IL. Mary Lawinger, MSW, is a social worker with Heartland Alliance in Chicago, IL. Anne Wildman, MSW is a School Social Worker in the State of Illinois.

Copyright ( 2018 Authors, Vol. 18 No. 3 (Spring 2018), 767-787, DOI: 10.18060/21650 
Egypt, Nigeria, Pakistan, and Iran represent 0.9 to 3.8\% of the Canadian immigration population respectively (Government of Canada, 2016; The Canadian Magazine of Immigration, 2017). Of this foreign-born population, nearly $20 \%$ are newcomer youth (Government of Canada, 2016). Although there is no single official definition, newcomer youth are typically defined as people between the ages of 12 and 24 who have been in Canada for fewer than five years (Affiliation of Multicultural Societies and Service Agencies of BC, 2016; Gouin, 2016; Ontario Council of Agencies Serving Immigrants, n.d.).

In contrast to native-born youth, newcomer youth in Canada (and throughout the world) face the challenges of behavioral and linguistic acculturation in their new country (Birman \& Morland, 2014; Bridging Refugee Youth \& Children's Services [BRYCS], 2010; MENTOR, 2009; Oberoi, 2016). Many immigrants (dubbed "newcomers" in this Canadian context) struggle with a new language, a new school system, and potentially new social norms (Birman \& Morland, 2014; BRYCS, 2010; MENTOR, 2009). Newcomer youth often undergo a process of acculturation that is very different from that of their parents, as they feel more pressure from peers to let go of their foreign identity and embrace an identity rooted in Canadian culture (Birman \& Morland, 2014; Oberoi, 2016). Additionally, many newcomer youth experience parent/child role reversals due to their superior understanding of the local language and need to help their parents in many of the daily tasks required to successfully navigate a new community (MENTOR, 2009; Oberoi, 2016).

These new pressures of acculturation and familial needs and expectations can cause significant stress and feelings of isolation among newcomer youth, resulting in increased difficulty relating to Canadian-born peers (Birman \& Morland, 2014; MENTOR, 2009), an increased risk of emotional distress in comparison to their peers (Hilario, Vo, Johnson, \& Saewyc, 2014), and specific stress related to exclusion, poverty, separation, and in some cases, trauma (MENTOR, 2009). Interventions focused on the unique needs of newcomer youth are critical in addressing the challenges and increased risks they face, and in supporting these youth in a healthy process of integration into their new communities.

\section{Conversation Club: A Group Mentoring Intervention for Canadian Newcomer Youth}

For Canadian newcomer youth, access to community services can serve as a protective factor in their migration and acculturation process (Canadian Pediatric Society, 2016). These community services can include: after-school programs, leadership development trainings, basic health trainings and advocacy, mental health services, dating violence advocacy programs, substance use treatment, employment resources, crime prevention resources, and summer camps. "Conversation Club" (also referred to here as "the Club") is a school-based group mentoring program developed by Big Brothers Big Sisters (BBBS) of Peel Province in response to the needs of diverse youth in the community. The program began in 2009 in partnership with local settlement organizations and libraries. The Club was quickly expanded to local schools and is now implemented in 14 sites, including high schools, libraries, community centers, and settlement agencies across Ontario. 


\section{Group Mentoring With Youth}

Group mentoring is defined by Kuperminc and Thomason (2014) as "natural or programmatic mentoring contexts in which one or more mentors work with at least two" mentees (p. 274). Mentees in group settings are free to try out new behaviors and to observe and learn from others' behaviors (Kuperminc \& Thomason, 2014). Additionally, group mentoring can allow for more culturally attuned work, as some individuals, particularly ethnic minority groups, prefer working with peers with similar experiences versus with a one-on-one mentor from another culture (Herrera, Vang, \& Gale, 2002; Kuperminc \& Thomason, 2014; Lindsay-Dennis, Cummings, \& McClendon, 2011; Utsey, Howard, \& Williams, 2003).

Group facilitators are expected to create an atmosphere of collaboration, introduce and maintain direction, promote positive change, and keep the focus of the group (Emelo, 2011; Kuperminc \& Thomason, 2014). Conversation Club facilitators, above all else, aim to meet the newcomer youth where they are and work to implement lessons that properly address the needs reported by youth in the program. This sense of collaboration is intended to fuel passion in the newcomer youth and empower them to maintain their ethnic identity while exploring Canadian culture and making new friends.

Group mentoring is one way to broadly address youth's needs by helping them connect with other newcomers, learn the new language and culture of Canada, and create their own sense of belonging and hope in their new environment. In order to do this, facilitators hear the experiences, hopes, and challenges of youth (MENTOR, 2009); create safe spaces of inclusion and belonging (MENTOR, 2009; Patton, Deutsch, \& Das, 2016); provide training in cultural competency (BRYCS, 2010; Oberoi, 2016) and conflict resolution (Deutsch, Wiggins, Henneberger, \& Lawrence, 2012); and involve youth with the program planning and shaping (BRYCS, 2010).

Due to their specific struggles, immigrants and refugees can experience significant benefits from mentoring (DuBois, Holloway, Valentine, \& Cooper, 2002; Rhodes, 1994). Specifically, group mentoring can provide a foundation for newcomer youth to relate to peers their age (Birman \& Morland, 2014; BRYCS, 2010; Cawood \& Wood, 2014; Crul \& Schneider, 2014; Deutsch, Reitz-Krueger, Henneberger, Ehrlich, \& Lawrence, 2016; Hilario et al., 2014; Schmidt, Morland, \& Rose, 2009) and receive peer and adult support through their adjustment process (Birman \& Morland, 2014). Mentees have a space to promote their bicultural identities (Morland, 2007) and find and foster positive relationships in their new environment (Birman \& Morland, 2014; Cawood \& Wood, 2014; Hilario et al., 2014; Oberoi, 2016; Schmidt et al., 2009).

The core tenets of group mentoring among youth are fostering connection, cohesion, and mutual support (Kuperminc \& Thomason, 2014). For these tenets to be achieved, safe spaces need to be formed in which culturally appropriate practices-including life skills, pro-social behaviors, critical thinking, and emotional coping - are promoted in alignment with specific cultural norms and expectations regarding adolescent development (Oberoi, 2016; Washington, Barnes, \& Watts, 2014). Especially among minority adolescents, group mentoring can create a sense of belonging (Cawood \& Wood, 2014) that might not be experienced in individualized mentorships. This solidarity with other students not only 
allows adolescents to relate with others going through similar experiences (i.e., adjusting to a new country), but provides them the opportunity to support one another and give advice to peers in times of distress (Jagendorf \& Malekoff, 2006). Creating these sincere connections with others helps to solidify youth's feelings of hope and belonging.

\section{Expansion and Dissemination of Youth Programs}

Although the value of group mentoring in the field is well established, there are many challenges which need to be addressed when disseminating and scaling up group mentoring programs for youth (Durlak \& DuPre, 2008; McIsaac, Read, Veugelers, \& Kirk, 2017). A common pitfall encountered in the dissemination process is that programs may be too complex for systems and providers to properly implement, which "may reduce adoption and diffusion of the program into systems" (Ozdemir \& Giannotta, 2014, p. 112). Researchers must focus on identifying the key elements of a program that make it effective in order to provide clear and simple guidelines for program facilitators that can be realistically implemented in provider settings (DuBois, Portillo, Rhodes, Silverthorn, \& Valentine, 2011; Rajan \& Basch, 2012). More quotidian factors such as inclement weather, school holidays, and lack of suitable locations can also have a potentially negative impact on the implementation of group mentoring programs for youth (Iachini, Beets, Ball, \& Lohman, 2014).

In order to implement beneficial group mentoring interventions with newcomer youth in Canada, there needs to be a clear purpose, an understanding of and planning for structural details (i.e., time and space), proper identification and/or creation of content that is appropriate and relevant for group goals, and, in many cases, support from schools and parents of the youth (BRYCS, 2010; Jagendorf \& Malekoff, 2006; Kurland, 1978; Malekoff, 1997). For adult and peer facilitators alike, it is essential for them to pay attention to the process, structure, and boundaries of the group, form alliances with parents, teachers, and school administrators, stress confidentiality and trust among group members and leaders, and make a demand for work (Jagendorf \& Malekoff, 2006). Efforts such as these, as reflected in this paper, help to contribute to impactful programming throughout expansion to new contexts and agencies.

\section{The Current Study}

Based on the program evaluation data from year one, we found that Conversation Club showed promise in addressing many of the important needs of its Canadian newcomers (Pryce, Kelly, \& Lawinger, 2018). Findings suggested improvement among participants, relative to a comparison group, on important metrics such as community belonging, ethnic and cultural identities, and hope for the future (Pryce et al., 2018). Despite the early promise of this intervention, the potential benefits of transferring these outcomes to newcomers in other settings had not been explored. Based on the initial findings, the Conversation Club creators sought to expand the Club to three sites in Ontario (i.e., Peel Region, York, and Ottawa), looking at this expansion from multiple methodological perspectives. 
In this mixed methods study, we sought to replicate our initial quantitative findings showing Conversation Club's promise as an intervention to positively impact newcomer youth's sense of belonging and hope. We also explored qualitatively how Conversation Club youth viewed their experience in the club intervention and in Canada more broadly. Finally, because this study focuses on Years Two and Three of the program evaluation, we report on focus group data with Conversation Club program staff regarding the utility of the Club's program manual and how to best scale the program up further.

\section{Procedures}

\section{Survey Data}

During the Fall semesters of two school years, (2014-15 and 2015-16), we worked with Conversation Club facilitators to secure consent from Conversation Club youth and families to conduct the study (total $n=149$ ). Based on the logic model developed with the Conversation Club program team, a questionnaire was developed to look at how the Conversation Club impacted newcomer youth's sense of hope and sense of belonging in their new country. These youth completed a questionnaire that asked for demographic data (age, gender, race/ethnicity, time already in Conversation Club, immigrant status) and also asked the youth about their sense of belonging in the Club and in Canada via the Perceived Cohesion Scale (PCS) (Cronbach's $\alpha=.96$; Bollen \& Hoyle, 1990) and overall sense of hope for the future via the Children's Hope Scale (CHS) (Cronbach's $\alpha=.83$; Snyder et al., 1997). These scales were part of the initial program evaluation year, as the dimensions of hope and belonging were identified by program staff as meaningful in considering the impact of the program on youth participants. Findings from Year One indicated that Conversation Club youth ( $\mathrm{n}=67$ ) had statistically significant changes on belonging and hope as compared to the comparison group $(n=25)$. The team elected to incorporate these measures into the program evaluation and expansion for Years Two and Three, reported here.

Data were collected by the Conversation Club team and support staff in each site, and transferred by post to the program evaluation team, who then entered the quantitative and demographic data into SPSS for later analysis.

\section{Interview and Focus Group Data}

Inclusion of qualitative methods reflects the effort, through this program evaluation, to understand the experience of the Conversation Club program from multiple perspectives. This mixed methods study employed methods sequentially (Creswell, 2013), which allows methods to be used as follow up from lessons learned from a prior method. In this case, the core research question was focused on the impact of Conversation Club participation on hope and belonging across multiple sites. The qualitative data collection allowed us to better understand what aspects of the Club facilitated belonging, and the process through which engagement in the Club takes place. While we assumed that the two methods would hold equal weight in the design, challenges to quantitative data collection, and the richness of the interview data generated, resulted in the qualitative data assuming more dominance in the study, a process noted often by experts in mixed methods (Creswell, 2013). 
At the conclusion of these two years, 18 interviews were conducted with a subset of Conversation Club participants across the three sites using a convenience sample. Eight of the interviewees were female and ten were male. The use of qualitative methods offered the flexibility to look at the experience of Club involvement from multiple viewpoints, taking into account environment and social context, while valuing the voice of participants across program levels (Malson, 2010).

These interviews were conducted by members of the Conversation Club team who were not facilitators of the youth's specific Club, so as to avoid any sense of coercion toward the interviewee. Interviewers were initially trained by a research team member on skills important to qualitative interviewing. This training involved several didactic sessions, as well as role plays using the interview protocol, and group feedback to the interviewer. Interviewers were then supervised by this same research team member through weekly group meetings focused on identifying challenges and uncertainties presented through the interview process. Interviewees were invited by program staff to participate in an interview, and a separate consent was obtained from parents for this piece of the research. Once consent was obtained, interviews took place in a private room separate from the Conversation Club during the same time as Club sessions. Interviews were audio recorded and transcribed for accuracy.

The analyses for this study were carried out in a team environment in which members engaged collaboratively to both challenge and validate emergent themes (Boyatzis, 1998; Lyons, 2007). An open coding method was first used by two of the paper's co-authors to capture initial themes. Following this initial coding, two of four co-authors individually reviewed all of the interview transcripts, using open coding to organize content into conceptual categories (Padgett, 2008) that reflected patterns and themes. These themes were organized into a larger outline that was elaborated on and challenged until consensus was reached. Finally, the two coders returned to the transcripts with a coding book and coded all transcripts.

Also, at the conclusion of Year Two, the two lead authors on the project co-facilitated a focus group with seven Conversation Club staff from three sites (three veteran staff with several experiences implementing Conversation Club, and four staff representing two of the new agencies adopting the program, who each had one year of experience implementing the Club). This focus group took place by phone and lasted 90 minutes. Content focused on the challenges and opportunities encountered by staff in their use of the Conversation Club manual and was based on a set of questions developed in collaboration by the lead authors and veteran program facilitators. As a structure for cofacilitation, one lead author asked the questions while the other kept notes and transcribed verbatim key insights offered by the focus group participants. Following the focus group, the two lead authors reviewed the notes and reflections together to identify themes around the manual and scaling up the intervention that are addressed later in this article. 


\section{Findings}

\section{Demographics}

Of the 149 Conversation Club members, 72 (48.5\%) were male and 76 (51\%) were female, and one student chose not to identify gender. While the group age ranged from 12 to 19 , the average age was 15 , and the average time the Conversation Club youth had been in Canada was 31.6 months. 85\% of the Conversation Club group members had Permanent Resident status in Canada, and the average time of involvement in the Club for this group was 10.25 months (this reflects the differences between the 2 new groups in York and Ottawa, and the long-standing Conversation Club groups in Peel). The Club members described themselves as coming from 33 different countries, including China, Syria, Egypt, Pakistan, the Philippines, \& India, making this the most diverse group of Conversation Club youth to date.

Table 1. Demographic distribution of Conversation Club participants

\begin{tabular}{l|c}
\hline \multicolumn{1}{c|}{ Demographics } & Participants \\
\hline Male n (\%) & $72(48.5 \%)$ \\
Female n (\%) & $76(51 \%)$ \\
Age Range & $12-19$ years \\
Average Age & 15 years \\
Avg. Months in Canada & 31.6 months \\
\% Permanent Residents & $85 \%$ \\
\hline
\end{tabular}

Findings noted in Table 2 reflect a within-group t-test performed with usable data from 2014-2016. Though the sample started with a total of 149 participants, several factors impacted study attrition, mostly due to students missing on the day of post-test, and some changing schools and leaving the group before the end of the program Thus, the sample was reduced to 105 and 114 for the two scales. Data from the within-group t-tests indicate that Conversation Club youth reported statistically significant change on their PCS scores (for both subscales of belonging and morale) and CHS scores (showing increased hope on both subscales in terms of both agency and pathways).

Table 2. Results of within-group t-tests for the treatment (Conversation Club) in Peel, York, and Ottawa 2014-2016

\begin{tabular}{lll|c|c|c|c|c|c|c}
\hline & & \multicolumn{3}{c|}{ Pre-Test } & \multicolumn{3}{c|}{ Post-Test } & \\
\cline { 3 - 9 } Subscale & Measure & $\mathrm{n}$ & Mean & St. Dev & $\mathrm{n}$ & Mean & St. Dev & t-test \\
\hline PCS & Belonging & 105 & 24.84 & 5.76 & 105 & 26.32 & 4.72 & $2.506^{*}$ \\
& Morale & 105 & 17.50 & 5.88 & 105 & 25.64 & 4.43 & $9.326^{*}$ \\
CHS & Agency & 114 & 13.88 & 2.8 & 114 & 14.94 & 2.57 & $3.794^{*}$ \\
& Pathways & 114 & 13.61 & 2.61 & 114 & 14.13 & 3.02 & $2.521^{*}$
\end{tabular}

Note. $*=\mathrm{p}<.025$ (Bonferroni Adjustment for each scale) $\mathrm{df}=104$ for PCS and $\mathrm{df}=113$ for CHS, based on usable pre-/post-tests. 
This data builds on data collected from Year One of this pilot evaluation, which also reflected significant improvement in sense of belonging and experience of hope among the program group relative to the comparison group (Pryce et al., 2018).

\section{Process Evaluation Data}

Participants in those two programs who were newly adopting Conversation Club were also asked to complete brief evaluations of their group sessions for the purpose of process evaluation. This involved rating the session on a scale of 1 (very bad) to 4 (very good; see Table 3), and responding to an open-ended query requesting the rationale for their rating, their favorite part of the session, and what could have been done to improve the session. Out of a total of 101 ratings by Conversation Club youth from October 2015-June 2016, a very strong picture emerged of the strengths of the Conversation Club program. $79.2 \%$ of the responses rated the Club sessions as 4 ("very good") and $12.9 \%$ of the remaining responses were in the "good" category, meaning that for these new sites, 92\% of the sessions were rated positively by the youth involved.

Table 3. Global ratings of program quality across sessions across new Conversation Club programs.

\begin{tabular}{l|c:c}
\hline Rating & $\begin{array}{c}\# \text { of } \\
\text { Responses }\end{array}$ & $\%$ \\
\hline 3 or 4 (Good or Very Good) & 94 & $92.1 \%$ \\
1 or 2 (Very Bad or Bad) & 7 & $7.9 \%$ \\
Total & 101 & $100.0 \%$ \\
\hline
\end{tabular}

When asked why they gave the session the rating they chose, the Conversation Club youth reported "I had fun" (48.5\%), "I liked playing games" (27.3\%), and "it made me feel good or happy" (8.9\%). The games the Club youth cited that involved using English and conversing with each other were frequently cited in their narrative descriptions, followed by the activities where they were encouraged to talk about their country of origin. Almost $50 \%$ of participants said a version of "nothing would make it better, it's great already," but there was a smaller group (30.9\%) who provided constructive feedback, stating that they wished for more active gym-based games, like volleyball and basketball, or more field trips.

\section{Qualitative Findings}

Across the eighteen interviews conducted with Conversation Club participants at the end of the program year, several themes emerged that assist in unpacking and illuminating the increased sense of belonging indicated by the quantitative data. The section below highlights some the most prominent themes. Of note, pseudonyms are used to protect the confidentiality of participants.

\section{Developing English Language Skills Increases Belonging}

Increased agility in speaking English, and related confidence and sense of belonging, emerged as the strongest themes in the interviews focused on the impact of Conversation 
Club. Daniel, an older adolescent from the Democratic Republic of the Congo, highlights some of this process in his reflection as associated with greater facility in speaking English, and related increased belonging and connection in relationships:

There's a lot of changes that I've seen and, I would say that one would be, uh, how to talk to people. Yeah. 'Cause for me, like, my first time here, I never talk to anyone. [...] So when I come, I join this group, they start teaching me good stuff which helps me, which gives me the word of discovering friends, of knowing how to be, ya know, nice to people. Yeah. So. I would say the key for me to talk to people, it was Conversation Club, because that's where I began.

Daniel's poignant reflection, which includes a reference to his anxieties as he navigates a new culture and environment, speaks powerfully of the "start" that Conversation Club gives him in "discovering friends" and "knowing how to be nice to people." This facilitated communication helped Daniel have an increased sense of belonging in Canada. Daniel's reflection is echoed by a large proportion of those interviewed; as comfort with English increased, so too did a perceived sense of connection, belonging, and confidence. As an example, Christine, from China, shares:

I think I might be more confident, because I was thinking, like, I am the English speaking not good. And now, I found someone is the same with me and everyone is the same. Everyone use the less greater words to talk about things. It make me feel better.

Samantha, a student from Jamaica, also reflects on the contribution of the Club to her ability to communicate and interact with others, sharing:

Now, it's like practicing how to talk to people and so when I go out into big society or bigger group, I can talk better each day and I can do my English more better. Because talking to you guys, it's like, I have to talk proper English, so it teaches me how to become better person and talk in proper English.

Aliyah expands again on this by linking this communication with self-improvement and connection with others. She states,

When you hear people talking in the language that you're not really familiar with, you start to learn. And you take chances, you start to talk to people, even though you're not perfect, but you get perfect. That's what I learned from Conversation Club.

This increased confidence with English supports Club participants not only in their sense of belonging in relationships, but in their feelings of competence in other spheres of life, such as classwork. Danny, an eighth grader who moved to Canada from Pakistan, outlines this clearly when she shares how building confidence through Conversation Club assisted in her school experience:

Conversation Club helped me bring up my confidence a little [...] I can talk in front of people about something I know, but, like, when you need to present, like, a presentation or anything and when it's all planned, that's when I start messing things up. Now it helped me when I'm presenting in class because in class I used 
to, like, my voice used to go down. I didn't even notice about that, my teachers are like, "Oh, yeah. That's getting better than it used to be."

Emmanuel, a second year high school student from India, continues this theme, affirming the impact of Conversation Club on his confidence, stating, "I learned that if you want to express yourself, you don't need to fear what others are thinking of yourself. Be confident and just say what you want to say. I have become more confident by joining this club.”

\section{Club Participation Increases Belonging}

One of the strongest themes that emerged from the interviews was in regards to the sense of belonging, and in turn, possibility that was associated with membership in Conversation Club. The majority of respondents discussed an initial sense of isolation and disorientation associated with relocation to Canada, and the important way that involvement in Conversation Club assisted them in having a sense of community. Emmanuel clearly expresses this experience of the Club:

This club is very nice; there is no discrimination. People don't judge you on the basis of your looks or your caste or your background. [...] Everyone is just, has this experience to share how they feel and they're encouraged to, like, express themselves, don't feel left out.

Jake, also a student from India, echoes this experience, sharing his sense of belonging as something that is "taught" through the Club, stating, "They talk equally. They always give us, like, a nice talk. They always teach us how to be, uh, how to be part in this group, and they always, uh, take care of us.”

A core aspect of this experience of belonging comes from the inclusion demonstrated by the Club. As mentioned by Emmanuel above, some of this inclusion is experienced by a lack of focus on "looks...caste, or your background". Rachel, an older adolescent student from the Democratic Republic of Congo, aptly describes this experience in her interview, stating, "When I joined Conversation Club, I feel like I'm not the only one, ya know. There are others and everyone is maybe interested in what I will say that or words or mistake, everyone like, people are interested in meaning, not mistakes.” This openness and lack of judgment can facilitate language, sharing, and, in turn, belonging. Christine, from China, takes this sense of acceptance and belonging even further, stating, "I felt this, like, a big family, and everyone is talking, and everyone is happy, and everyone is friendly and kind, and that is it.” Aliyah highlights this sense of family as beyond her daily experience of a traditional classroom, sharing:

In the classroom, it's like each and every individual, they just come to class with themselves and leave. Conversation Club is like a family. Everyone has a responsibility for others, everyone has some kind of connection with others, they respect each other. That is the best thing, that's the thing I love the most.

Here, Aliyah touches on the fact that many Conversation Club participants come from countries where the dominant culture is more collectivistic than that in Canada. Migration from a collectivistic to an individualistic culture poses difficulties for immigrants, particularly for youth who must navigate between the cultures of their parents and their 
new home (Schwartz et al., 2015). Although immigrant youth typically undergo significant acculturation, some research indicates that underlying values, such as collectivism, do not change significantly with the acculturation process (Rosenthal, Bell, Demetriou, \& Efklides, 1989). Conversation Club provides participants with an opportunity to learn about and engage with Canadian culture in a close-knit group setting which promotes feelings of familiarity and comfort.

Beyond feeling a sense of belonging to a group, many interviewees expressed a sense that such a belonging translates to feeling a part of the society as a whole. Marva, who came to Canada from India, makes this connection beautifully, stating, "It's made me feel like I'm no different than the others and that I don't have to change to fit into society and stuff.” Joe, from Pakistan, expands this further, linking his participation in the Club with a greater sense of being a part of Canada. He states, "It is a community, right, and community is a part of Canada. So, like in Canada, there is a lot of people from other countries, so, but we stick together. We feel like we are in Canada." Manish and others echo this sentiment, stating, "Of course, I feel Canadian. 'Cause when I came here, I was feeling something away from the people here. But Conversation Club and other my friends, everyone, made me to be connected to Canada. Now I feel like a Canadian.” Many Conversation Club members appear to experience this increased sense of belonging through Conversation Club as an integral part of their development of identity as newcomers and Canadians.

\section{Interpersonal Support Increases Belonging}

Participants shared that the sense of belonging also comes from the increased social support and connection that all interviewed youth shared when reflecting on the impact of the Club. This support was provided through connection to peers, as well as Club facilitators and volunteer mentors. Below is one of many examples, as articulated by Daniel, of the experience of friendship and connection cultivated through the Club:

Like right now, I can't count how many friends I have because there are many. So Conversation Club helped me know how to talk to people, how to know people.

Whenever I walk in the hallway, I talk to them even though I don't know them.

The abundance of connections that Daniel references is in stark contrast to the initial isolation experienced by many Conversation Club participants as expressed earlier in this section.

Connection extends beyond that with peers to program staff and mentors in the group, as expressed by students in referencing the role of program facilitators and mentors in the experience of building relationships through the program. The participants' words exemplify the importance of these relationships in helping them feel included and empowered in the Club. As James shares,

They'll just talk and help you, they'll ask about school, they'll talk more about their experience when they were at school. And then, ya know, they'll just relate it and try to help you and give you advice and stuff. 
Laura connects this experienced "friendliness" with an increase in energy that she has each day the Club is scheduled. In referencing her experience of Conversation Club mentors, she shares:

They're friendly. They won't be like, "I don't want to help you." They help us. Like, like a sister or a brother, like that. So I never skip the Conversation Club. [...] When it's Thursday, I feel, like, an energy. When it's 3:30, "Oh! Conversation Club! I need to go!" Then I take every step and go fast. And if I see them, I feel happy.

Laura shares the support that she receives, "like a sister or a brother." She translates that support to her enthusiasm for Conversation Club and making sure she attends as much as possible. This suggests that Conversation Club has the potential to overcome the common challenge of adolescent participation in after school activities by providing social support that participants do not find elsewhere.

Manish also articulates the unique support provided by facilitators and mentors, and the evolving role that they serve in his life. Manish reflects,

At first I joined Conversation Club, [staff] was like a teacher to me. But now, she's more than a teacher, she's a friend to me. She just tells what is good and what is bad. And it's [Conversation Club] better than other organizations because my mentor, like, she is really good, she never yells at us. So it makes other students to feel comfortable and better.

For Manish, the friendly, accepting, advising support offered by Conversation Club facilitators and mentors set the program apart from other after school opportunities. The increased sense of belonging and safety from these adults translates to an increased investment in Conversation Club, as well as well-being and support.

\section{Focus Group Data}

To further inform our understanding of what works within Conversation Club, and what needs to be adjusted or changed in order to further support its dissemination, we were able to complement our mixed method data by facilitating a focus group with Club staff (n = 7; all female). This conversation was largely focused on challenges faced by staff in making use of the Conversation Club manual, a tool that had been developed for their use in the early phases of program dissemination. Anecdotally, we were aware that staff were making minimal use of the manual (i.e., "I found the manual gives a few suggested ideas for activities and things to do during the club, but it's more of almost a background before you even get to the Club"), and yet that the larger mentoring organization was hoping to build off of this tool for further dissemination. However, our understanding was limited as to what was needed to make this manual more usable.

Through our focus group conversation, we were able to develop greater understanding in this area. First, we explored manual use to date more systematically. Conversation Club leaders reported using the manual mostly to orient themselves to the general goals and scope of the program, and to develop initial start-up activities (e.g., "I used the manual at the beginning of the session, but then as I got going, I didn't really refer to it from there on 
out”). They all reported that as written, the Conversation Club manual was not useful as an ongoing resource for regular consultation or for guidance in confronting challenges.

Next, our focus group addressed these limitations. Participants were asked how additional programs within Canada could adopt Conversation Club in a way that is faithful to its original mission, as well as being likely to promote the benefits that have been documented. According to the professionals participating in the focus group, the manual needed to be divided into broad phases that reflected the natural ebbs and flows of the Club, including the initial phase (i.e., first month), middle phase, and culminating phase (i.e., last two months). They thought that these phases should be outlined and structured within a revised manual, using specific examples and suggestions from the sites currently implementing Conversation Club. According to focus group participants, these phases reflected the development of the group, from introduction, development, engagement, and the development of co-leadership with the Conversation Club youth (Forsyth, 2018; Northern \& Kurland, 2001; Yalom, 1995).

Within each phase, participants proposed having a checklist that would help new Conversation Club groups implement the Club with fidelity. This checklist needed to not only highlight important pieces, but also reflect practice wisdom developed through the pilot of Conversation Club across three regions. This needed to be sequential and structured in such a way that new facilitators are incentivized, at least structurally, to revisit the checklist within each phase.

In addition to structural changes, participants highlighted the importance of "shared authorship" as a core value in working with Conversation Club youth, and one that needs to be outlined up front in a manual, as well as woven throughout suggested exercises and foci. According to participants, newcomer youth must have a voice in co-creating a program such as Conversation Club. As an example, one participant said, "They [youth] would see you as the leader, but there is that understanding that the mentors and the youths themselves are able to bring forward their own ideas and the direction of where they want their program and activities to go.” Lessons learned over the last year confirm how important it is to both call for and demonstrate examples of how newcomer youth shared authorship works within Conversation Club, and how essential it is to the success of the program.

Finally, as is well supported in evidence-based work on the dissemination of programs (Bernal, Jiménez-Chafey, \& Domenech Rodríguez, 2009; Kelly, Raines, Stone, \& Frey 2010; Taussig et al., 2015), it is critically important to identify how and where new Conversation Club groups can tailor their work to their specific contexts as they adopt this exciting program. One participant summarized this by saying, "I tried to go along with activities [per the manual], but I found a lot of the youth were not really enjoying that...so informing a new staff member to remain flexible and just get the input of the youth would be very important." The work on Conversation Club to date shows that having a basic structure for the phases of the program, with myriad opportunities for Conversation Club group mentor leaders to tailor the specific activities in each phase to the specific cultural contexts of the participating Club youth, appears to have found a balance between keeping program fidelity while still allowing for adaptability and "shared voice" with Conversation 
Club youth. Conversation Club group leaders can add their own unique contributions, particularly by including youth voice in the creation and implementation of the Club tasks and activities. Our focus group data indicates that Conversation Club facilitators say they need some guidance in order to balance the important Conversation Club program components that will assure program fidelity with their own creative ideas in engaging youth voice.

\section{Discussion and Implications for Research and Social Work Practice}

This paper reinforces findings from our initial study (Pryce et al., 2018) and further situates Conversation Club within the group mentoring literature as a promising intervention that merits further investigation, particularly for immigrant youth and adolescents (Birman \& Morland, 2014). With its focus on creating a consistent, safe, and interactive space for newcomer youth, Conversation Club appears to support immigrant youth who often find themselves in a critical and risky transition. Based on the quantitative and qualitative data described here, Conversation Club demonstrates strong potential as an intervention that can build newcomer youth's sense of hope and sense of belonging to their new home. It is also of note that these outcomes, which were embedded in the initial logic model for this evaluation, were informed largely by the practice wisdom and expertise of the Conversation Club group leaders themselves and their creative application of the core ideas of Conversation Club within their specific contexts.

The findings noted above have significant implications for social work practice. Research in the area of program dissemination indicates that programs facilitated by social workers need to be flexible and adaptable (Ozdemir \& Giannotta, 2014) to allow facilitators "to modify a program to make it more effective in a specific context" (Durlak \& DuPre, 2008, p. 341). In order to achieve this, researchers must "identify critical program elements” (DuBois et al., 2011, p. 79) so that energy can be devoted to ensuring that those elements are maintained, while offering facilitators clear guidelines as to where adaptation of the curriculum is and is not desirable. This also speaks to the importance of training social work practitioners and implementers of interventions such as Conversation Club in how to adhere to a manual for an intervention while remaining flexible in addressing the specific needs of individual participants. Specific to Conversation Club, moving forward with the dissemination, it will be critical to utilize facilitator feedback in continuing to revise the manual to maximize program flexibility and fidelity.

Newcomers often face social, emotional, academic, and personal challenges as they seek to adjust to a new culture and language (Gouin, 2016; Hilario et al., 2014; Oberoi, 2016). Particularly given the unique relational needs of adolescents, the results of this intervention suggest the Conversation Club is offering much that these young people report to seek and value, particularly as they build on the adolescent's need to find productive connections and engage in "shared voice" activities. It is important to note that this study indicates that group mentoring programs may be more effective for newcomer youth than would be individual mentoring programs. This possibility warrants further exploration in both research and practice settings. 
Results from this phase of dissemination of this promising program are encouraging for several reasons. In these two years, we saw significant and similar gains from pre- to post-test for all 3 regions on both the Hope Scale and Perceived Cohesion Scale. Significantly, this experience of hope and belonging appears to be manifest across youth migrants of 33 different countries, some of which represent nation states that maintain ongoing and persistent tensions (e.g., India/Pakistan, Syria/Egypt). Strikingly, Conversation Club appears to be a place where that tension is absent. In its place is the clear formation of a new community and one that appears to be able to celebrate ethnic differences while also emphasizing the newcomer youth's transition to becoming part of Canada.

In summary, it is clear that the Conversation Club program has significant promise in impacting key adolescent outcomes of belonging and hope across 3 different regions of Ontario. This initial data focused on dissemination suggests that Conversation Club is a program that can be disseminated to additional mentoring settings. Conversation Club appears to be able to engage and build on the strengths of young adolescents as well as young adult mentors preparing in engaging in supportive, collaborative relationships that enhance identity and development. These connections, between peers, mentors, and even to their homeland, offer a powerful sense of belonging and membership for these adolescents. As Conversation Club seeks to expand further in Canada, and as others work to address the myriad needs and strengths of migrant and refugee adolescents, is it critical to maintain attention to the adaptability and flexibility of the Conversation Club program that has made it successful for many diverse young people.

\section{Limitations}

While this study shows some positive outcomes, there are, as always, important limitations to bear in mind in interpreting results. The sampling plan represented a convenience sample of the youth that agreed to participate across three regions and does not include any comparison groups that could more fully show the impact of Conversation Club on its participants. From the quantitative component of the study, there was missing data (i.e., roughly 30-40 youth out of a total of 149 had missing data for some questions at post-test), and that missing data requires that the quantitative findings be interpreted with much caution; while not uncommon for a front-line program attempting to do an independent program evaluation in collaboration with our research team, this is still important to note. Additionally, a significant limitation for the entire study (and indeed, the Conversation Club intervention overall) is the informal nature of how English language comprehension is evaluated. Although this project took place in close partnership with program developers and staff, all of whom had history implementing the Club with young people in their communities, all referrals for the program were made by schools and teachers in the English Language Learner (ELL) classroom. Researchers and the Conversation Club team relied on that assessment to establish how much English the participants had and their capacity to engage in the program and research protocol. Future research needs to formally include a measure of English proficiency to enhance the strength of potential outcome findings. Additional research is needed more broadly on Conversation 
Club, as well, both in terms of a larger sample in the Club groups as well as having a comparison group, preferably one that is randomly assigned to a control condition.

Although this project benefits from qualitative data from two perspectives, including Conversation Club participants and facilitators, voluntary interviews at one time point provide a limited view into the experience of the Club. Further, given that this project served as an evaluation of the program and an exploration of considerations for dissemination, the focus of the interview and focus groups, while building on one another, do not serve to deepen the qualitative knowledge provided through this project. More depth in qualitative inquiry, perhaps using phenomenology or case study method, is merited in order to more deeply understand the meaning and experience available to immigrant and migrant youth through group-based mentoring programs. Ensuring additional rigor through interpretation or other means (such as assessment of inter-rater reliability between coders) could also enhance this work.

\section{Conclusion}

This mixed-method program evaluation continues to reveal encouraging findings that invite additional investigation into the potential scope and reach of Conversation Club to other areas of Canada. This work represents strong collaboration from a dedicated set of practitioners and researchers and continues to spring from a "shared authorship" approach built on the wisdom of many years of building this innovative effort to incorporate mentoring into services for migrant and refugee adolescents. Future work would do well to further investigate the ways by which Conversation Club can be feasibly and effectively translated to additional regions and settings, as well as ways by which membership in the Club may impact more broadly the adjustment of newcomer Canadian adolescents across this critically important developmental stage.

\section{References}

Affiliation of Multicultural Societies and Service Agencies of BC [AMSSA]. (2016). Newcomer youth: Challenges and strengths. Retrieved from http://www.amssa.org/resources/migrationmatters-info-sheet-series/.

Bernal, G., Jiménez-Chafey, M. I., \& Domenech Rodríguez, M. M. (2009). Cultural adaptation of treatments: A resource for considering culture in evidence-based practice. Professional Psychology: Research and Practice, 40(4), 361-368. doi: https://doi.org/10.1037/a0016401

Birman, D., \& Morland, L. (2014). Immigrant and refugee youth. In D. L DuBois \& M. J. Karcher (Eds.), Handbook of Youth Mentoring (pp. 273-289). Los Angeles: Sage. doi: https://doi.org/10.4135/9781412996907.n24

Bollen, K. A., \& Hoyle, R. H. (1990). Perceived cohesion: A conceptual and empirical examination. Social Forces, 69(2), 479-504. doi: https://doi.org/10.1093/sf/69.2.479

Boyatzis, R. E. (1998). Transforming qualitative information: Thematic analysis and code development. Los Angeles: Sage Publications. 
Bridging Refugee Youth \& Children's Services [BRYCS]. (2010). New directions in mentoring refugee youth. Retrieved from http://www.brycs.org/documents/upload/BRYCS-BRIEF-Mentoring-Summer2010.pdf

Canadian Pediatric Society. (2016). Community resources for immigrant and refugee youth. Retrieved from https://www.kidsnewtocanada.ca/health-promotion/youthresources

Cawood, N. D., \& Wood, J. M. (2014). Group mentoring: The experience of adolescent mentees on probation. Social Work with Groups, 37(3), 213-229. doi: https://doi.org/10.1080/01609513.2013.862895

Creswell, J. W. (2013). Research design: Qualitative, quantitative, and mixed methods approaches. Thousand Oaks: Sage Publications.

Crul, M., \& Schneider, J. (2014). Mentoring: What can support projects achieve that schools cannot? Retrieved from SIRIUS Network Policy Briefs series [Issue 2] http://www.sirius-migrationeducation.org/wpcontent/uploads/2015/02/SIRIUS_Mentoring_FINAL.pdf.

Deutsch, N. L., Reitz-Krueger, C. L., Henneberger, A. K., Ehrlich, V. A. F., \& Lawrence, E. C. (2016). "It gave me ways to solve problems and ways to talk to people." Outcomes from a combined group and one-on-one mentoring program for early adolescent girls. Journal of Adolescent Research, 32(3), 291-322. doi: https://doi.org/10.1177/0743558416630813.

Deutsch, N. L., Wiggins, A. Y., Henneberger, A. K., \& Lawrence, E. C. (2012). Combining mentoring with structured group activities: A potential after-school context for fostering relationships between girls and mentors. The Journal of Early Adolescence, 33(1), 44-76. doi: https://doi.org/10.1177/0272431612458037.

DuBois, D. L., Holloway, B. E., Valentine, J. C., \& Cooper, H. (2002). Effectiveness of mentoring programs for youth: A meta-analytic review. American Journal of Community Psychology, 30(2), 157-197. doi: https://doi.org/10.1023/A:1014628810714

DuBois, D. L., Portillo, N., Rhodes, J. E., Silverthorn, N., \& Valentine, J. C. (2011). How effective are mentoring programs for youth? A systematic assessment of the evidence. Psychological Science in the Public Interest, 12(2), 57-91. doi: https://doi.org/10.1177/1529100611414806.

Durlak, J. A., \& DuPre, E. P. (2008). Implementation matters: A review of research on the influence of implementation on program outcomes and the factors affecting implementation. American Journal of Community Psychology, 41, 327-350. doi: https://doi.org/10.1007/s10464-008-9165-0.

Emelo, R. (2011). Group mentoring best practices. Industrial and Commercial Training, 43(4), 221-227. doi: https://doi.org/10.1108/00197851111137898.

Forsyth, D. R. (2018). Group dynamics. Boston, MA: Cengage Learning. 
Gouin, R. (2016). Facilitating the integration of newcomer children and youth: Study on the government's initiative to resettle Syrian refugees to Canada. Retrieved from https://www.bgccan.com/wp-content/uploads/sites/3/2017/03/BGCC-briefCitizenship andImmigration-2016.pdf

Government of Canada. (2016). \#WelcomeRefugees: Key figures. Retrieved from https://www.canada.ca/en/immigration-refugeescitizenship/services/refugees/welcome-syrian-refugees/key-figures.html

Government of Canada. (2017). Supplementary information 2018-2020 immigration levels plan. Retrieved from https://www.canada.ca/en/immigration-refugeescitizenship/news/notices/supplementary-immigration-levels-2018.html

Herrera, C., Vang, Z., \& Gale, L. Y. (2002). Group mentoring: A study of mentoring groups in three programs [Report]. Retrieved from https://files.eric.ed.gov/fulltext/ED467570.pdf

Hilario, C. T., Vo, D. X., Johnson, J. L., \& Saewyc, E. M. (2014). Acculturation, gender, and mental health of Southeast Asian immigrant youth in Canada. Journal of Immigrant and Minority Health, 16(6), 1121-1129. doi: https://doi.org/10.1007/s10903-014-9978-X.

Iachini, A. L., Beets, M. W., Ball, A., \& Lohman, M. (2014). Process evaluation of "Girls on the Run": Exploring implementation in a physical activity-based positive youth development program. Evaluation and Program Planning, 46, 1-9. doi: https://doi.org/10.1016/j.evalprogplan.2014.05.001

Jagendorf, J., \& Malekoff, A. (2006). Groups-on-the-go: Spontaneously formed mutual aid groups for adolescents in distress. Social Work with Groups, 28(3-4), 229-246. doi: https://doi.org/10.1300/J009v28n03_15

Kantor, J., \& Einhorn, C. (2016). Refugees encounter a foreign word: Welcome. The New York Times. Retrieved from http://www.nytimes.com/2016/07/01/world/americas/canada-syrian refugees.html

Kelly, M. S., Raines, J. C., Stone, S., \& Frey, A. (2010). School social work: An evidence-informed framework for practice. Oxford, UK: Oxford University Press. doi: https://doi.org/10.1093/acprof:oso/9780195373905.001.0001.

Kuperminc, G. P., \& Thomason, J. D. (2014). Group mentoring. In D. L DuBois \& M. J. Karcher (Eds.), Handbook of Youth Mentoring (pp. 273-289). Los Angeles: Sage. doi: https://doi.org/10.4135/9781412996907.n18.

Kurland, R. (1978). The neglected component of group development. Social Work with Groups, 1(2), 173-178. doi: https://doi.org/10.1300/J009v01n02 06.

Lindsay-Dennis, L., Cummings, L., \& McClendon, S. C. (2011). Mentors' reflections on developing a culturally responsive mentoring initiative for urban African American girls. Black Women, Gender \& Families, 5(2), 66-92. doi: https://doi.org/10.5406/blacwomegendfami.5.2.0066. 
Lyons, E. (2007). Analysing qualitative data: Comparative reflections. In E. Lyons \& A. Coyle (Eds.), Analysing qualitative data in psychology (pp. 158-173). London, U.K: Sage Publications Ltd. doi: https://doi.org/10.4135/9781446207536

Malekoff, A. (1997). Planning in group work: Where we begin. Group Work with Adolescents: Principles and Practice (pp. 53-80). New York: The Guilford Press.

Malson, H. (2010). Qualitative methods from psychology. In I. Bourgeault, R. Dingwall, \& R. de Vries (Eds.), The Sage Handbook of Qualitative Methods in Health Research (pp. 193-211). Los Angeles: Sage. doi: https://doi.org/10.4135/9781446268247.n11

McIsaac, J. D., Read, K., Veugelers, P. J., \& Kirk, S. F. (2017). Culture matters: A case of school health promotion in Canada. Health Promotion International, 32, 207-217. doi: https://doi.org/10.1093/heapro/dat055

Metcalfe-Hough, V. (2015.). The migration crisis? Facts, challenges and possible solutions. Retrieved from https://www.odi.org/sites/odi.org.uk/files/odiassets/publications-opinion-files/9913.pdf.

MENTOR. (2009). Mentoring immigrant \& refugee youth: A toolkit for program coordinators. Retrieved from https://files.eric.ed.gov/fulltext/ED522073.pdf

Migration Policy Institute. (2015). International migration statistics. Retrieved from http://www.migrationpolicy.org/programs/data-hub/international-migration-statistics

Morland, L. (2007). Promising practices in positive youth development with immigrants and refugees. The Prevention Researcher, 14(4), 18-21.

Northen, H., \& Kurland, R. (2001). Social work with groups. NY: Columbia University Press.

Oberoi, A. (2016). Mentoring for first-generation immigrant and refugee youth. National Mentoring Resource Center. Retrieved from http://nationalmentoringresourcecenter.org/images/PDF/ImmigrantRefugeeYouth_P opulation_Review.pdf

Ontario Council of Agencies Serving Immigrants. (n.d.). Who is an immigrant, refugee, newcomer, \& undocumented person? Retrieved from http://www.newyouth.ca/immigration/settlement-services/what-immigrant-refugeenewcomer-undocumented-person.

Ozdemir, M., \& Giannotta, F. (2014). Improving dissemination of evidence-based programs through researcher-practitioner collaboration. New Directions for Child and Adolescent Development, 141, 107-116. doi: https://doi.org/10.1002/yd.20090.

Padget, D. (2008). Qualitative methods in social work research. Thousand Oaks, California: Sage Publications, Inc.

Patton, C. L., Deutsch, N. L., \& Das, A. (2016). Coordination, competition, and neutrality autonomy and relatedness patterns in girls' interactions with mentors and peers. The 
Journal of Early Adolescence, 36(1), 29-53. doi: https://doi.org/10.1177/0272431614556349

Pryce, J. M., Kelly, M. S., \& Lawinger, M. (2018). Conversation Club: A Group Mentoring Model for Immigrant Youth. Youth \& Society, 00(0), 1-21 [online first]. doi: https://doi.org/10.1177/0044118X18780526.

Rajan, S., \& Basch, C. E. (2012). Fidelity of after-school program implementation targeting adolescent youth: Identifying successful curricular and programmatic characteristics. Journal of School Health, 82, 159-165. doi:

https://doi.org/10.1111/j.1746-1561.2011.00681.X.

Rhodes, J. E. (1994). Older and wiser: Mentoring relationships in childhood and adolescence. Journal of Primary Prevention, 14(3), 187-196. doi: https://doi.org/10.1007/BF01324592

Rosenthal, D. A., Bell, R., Demetriou, A., \& Efklides, A. (1989). From collectivism to individualism? The acculturation of Greek immigrants in Australia. International Journal of Psychology, 24, 57-71. doi: https://doi.org/10.1080/00207594.1989.10600032.

Schmidt, S., Morland, L., \& Rose, J. (2009). Growing up in a new country: A positive youth development toolkit for working with refugees and immigrants. Retrieved from the BRYCS website http://www.brycs.org/documents/upload/GrowingUpInANewCountry-Web.pdf

Schwartz, S. J., Zamboanga, B. L., Mason, C. A., Baezconde-Garbanati, L., Des Rosiers, S. E., Villamar, J. A., ... Szapocznik, J. (2015). Developmental trajectories of acculturation: Links with family functioning and mental health in recent-immigrant Hispanic adolescents. Child Development, 86(3), 726-748. doi: https://doi.org/10.1111/cdev.12341.

Snyder, C. R., Hoza, B., Pelham, W. E., Rapoff, M., Ware, L., Danovsky, M., ... \& Stahl, K. J. (1997). The development and validation of the Children's Hope Scale. Journal of Pediatric Psychology, 22(3), 399-421. doi: https://doi.org/10.1093/jpepsy/22.3.399

Statistics Canada. (2011). National household survey profile, 2011. Retrieved from https://www12.statcan.gc.ca/nhs-enm/2011/dp-pd/prof/index.cfm?Lang=E

Taussig, H., Weiler, L., Rhodes, T., Hambrick, E., Wertheimer, R., Fireman, O., \& Combs, M. (2015). Fostering healthy futures for teens: Adaptation of an evidencebased program. Journal of the Society for Social Work and Research, 6(4), 617-642. doi: https://doi.org/10.1086/684021

The Canadian Magazine of Immigration. (2017). Canada: Immigrants by source country2016. Retrieved from http://canadaimmigrants.com/canada-immigrants-bysource-country-2016/. 
Utsey, S. O., Howard, A., \& Williams III, O. (2003). Therapeutic group mentoring with African American male adolescents. Journal of Mental Health Counseling, 25(2), 126-139. doi: https://doi.org/10.17744/mehc.25.2.q3wda06r0ul0x97c

Vieira, P. (2015, November 24). Canada outlines plans to bring in 25,000 Syrian refugees; Ottawa will bring in Syrians in two groups, even as other nations express concerns about accepting refugees. Retrieved from Wall Street Journal [Online] website https://www.wsj.com/articles/canada-outlines-plans-to-bring-in-25-000syrian-refugees-1448402154

Washington, G., Barnes, D., \& Watts, R. J. (2014). Reducing risk for youth violence by promoting healthy development with pyramid mentoring: A proposal for a culturally centered group mentoring. Human Behavior in Social Environment, 24(6), 646-657. doi: https://doi.org/10.1080/10911359.2014.922789

Yalom, I. D. (1995). The theory and practice of group psychotherapy. NY: Basic Books (AZ).

Author note: Address correspondence to: Julia Pryce, PhD, School of Social Work, Loyola University Chicago, 1 E. Pearson, \#544, Chicago, IL. 60611. jpryce@luc.edu. 